\title{
Energy-Efficient Power/Rate Control and Scheduling in Hybrid TDMA/CDMA Wireless Sensor Networks*
}

\author{
Tao Shu and Marwan Krunz ${ }^{\dagger}$ \\ Department of Electrical and Computer Engineering \\ University of Arizona \\ Tucson, AZ 85721, USA \\ Email: $\{$ tshu, krunz\}@ece.arizona.edu
}

\begin{abstract}
We consider a hybrid TDMA/CDMA wireless sensor network (WSN) and quantitatively investigate the energy efficiency obtained by combining adaptive power/rate control with time-domain scheduling. The energy efficiency improvement is carried out with respect to interfering-cluster scheduling, intra-cluster node scheduling, and transmission powers and times (durations) control (PTC) for individual nodes. The interfering-cluster scheduling is formulated as a vertex-coloring problem, which can be solved efficiently using existing numerical algorithms in graph theory. For the node scheduling problem, we present a heuristic algorithm, which iteratively searches for the best schedule in such a way that the energy consumption keeps decreasing after every iteration. Compared with the exponentially complicated exhaustive search algorithm, this heuristic algorithm has polynomial computing complexity and can provide optimal solutions in the most simulated cases. For the transmission power/time control, two simplified PTC schemes, namely, PTC-UT and PTC-USG, are proposed and studied based on our previous optimization work PTC-IPT. We show that PTC-UT and PTC-USG provide comparable energy efficiency to PTC-IPT at only half of its complexity. Numerical examples are used to validate our findings.
\end{abstract}

Keywords: Hybrid TDMA/CDMA, wireless sensor network, joint power and time control, scheduling, convex optimization.

\section{Introduction}

In recent years, we have witnessed an increased interest in using wireless sensor networks (WSNs) in a wide range of military and civilian applications [1]. To lower the cost, in these networks sensors are typically powered by non-rechargeable batteries. Once deployed, the sensors in the field are usually left unattended, making the replacement of the batteries impractical

*Part of this work was presented at the IEEE GLOBECOM 2006 Conference, San Francisco, Dec. 2006.

$\dagger$ Contact author. 
(if not impossible). To provide long-lasting operation time, energy-efficient system architecture and communication protocols are crucial to the successful deployment of WSNs.

In this paper, we are interested in improving the energy efficiency of a large-scale WSN that may contain thousands of nodes. Systems at this scale are expensive, and thus it is more desirable to make their operation last long. Due to the extremely large amount of nodes in the network, the collision between nodes becomes more severe, making the interference between simultaneous transmissions a major factor in deteriorating the system's energy efficiency. Thus a good medium access control (MAC) algorithm is needed to coordinate the transmissions of different nodes in such a way that the interference between nodes can be minimized. When transmission delay is concerned, this problem becomes challenging, because directly applying the conventional contention-based or TDMA-based MAC design to a large scale WSN generally leads to either poor collision performance or large transmission delay. This difficulty is further elaborated below.

A contention-based MAC design intends to take advantage of the low duty-cycle communication between sensors. In these protocols, a sensor turns off its radio during idle times or when other nodes are transmitting to avoid unnecessary energy consumption. It turns on its radio to access the channel only when it needs to transmit. A notable example of such MAC protocols is the SMAC proposed in [3], which is based on the carrier sensing multiple access (CSMA) technology. SMAC forces sensor nodes to operate at a fixed duty cycle by putting them into periodic sleep during their idle time. To make sleep period adaptable to variable traffic-load conditions, adjustable duty cycle was introduced in the TMAC [4] and PMAC [5] protocols, where the sleep-wake-up schedules are adaptively determined by the activity or the traffic pattern of the neighboring nodes, respectively. The performance of these CSMA-based MAC protocols degrades significantly when node population is large, due to the notorious "hidden terminal" problem, i.e., collision on packet transmissions can happen in any two-hop neighborhood of a node. The conflicting packets need to be retransmitted, thus the energy and time on transmitting previous copies are wasted, leading to deteriorated energy efficiency.

On the other hand, time division multiple access (TDMA) has a built-in advantage of eliminating the hidden terminal problem without any overhead. This is achieved by scheduling the transmission of neighboring nodes into different (non-overlapping) time slots. In addition, TDMA also has an embedded "sleep" characteristics: A radio can be naturally turned on and 
off according to the allocated slot schedule. These desirable features make TDMA a natural choice for MAC protocols in small to medium-sized WSNs. Many TDMA-based MAC protocols have been proposed in the literature, some of which have been standardized, e.g., the LEACH protocol in [2], the IEEE 802.15.1 Bluetooth [8], and the IEEE 802.15.4 ZigBee [9]. Despite all these good features, however, one major disadvantage of TDMA is its scalability. For example, more slots in each frame are needed when the sensor population increases, leading to longer transmission delay for each node. To accommodate more sensors in each frame, the length of a slot also needs to be shrinked, leading to more rigid synchronization requirement between sensors. At the same time, TDMA has a low utilization of slots when the network size is large. For event-driven applications, not every node will have data to transmit in every frame. Therefore, although a lot of slots have been allocated for senors, not all of them will be used in each frame. Those allocated but unused slots could have been used by those active nodes, making their transmission rates smaller, and thus saving more energy on communication [12]. Similar problem happens when the number of sensors changes. It is not easy for a TDMA protocol to dynamically change its frame length and time slot assignment to accommodate topology changes.

To tackle these difficulties and make TDMA more efficient under larger network size, some renovations on TDMA have been made. The work in [6] proposes the combined CSMA/TDMA Z-MAC protocol, which acts in CSMA mode when the traffic load is light, and transfers to TDMA mode under heavy traffic load. The algorithm of Group TDMA is proposed in [7], which partitions nodes into disjoint non-interfering sets of transmitters and receivers, and allocates different slots to different sets. Under large node population, Group TDMA still needs a lot of slots in each frame. The bit-map-assisted MAC (BMA-MAC) proposed in [13] improved upon LEACH by partitioning a frame into two segments, i.e., data transmission segment and idle segment, and the boundary between them is adjustable. Only those nodes that have data to send in the current frame will be allocated with a dedicated slot in the data transmission segment. The analytical work in [14] and [15] further improved upon [13] by showing that the ultimate energy-optimal transmission under TDMA requires variable slot lengths for different nodes in each frame. Let alone the difficulty of the extremely strict synchronization among sensors in order to align slots of variable length together, the complexity of the algorithm, which decides the optimal slot lengths for individual sensors, grows exponentially with the number of active nodes. Due to the poor scalability, currently TDMA-based MAC design is 
only adopted by small-scale systems, i.e., a Bluetooth [8] cluster contains at most 8 nodes, and a ZigBee [9] cluster supports at most 7 nodes.

In this paper, we propose a hybrid TDMA/CDMA architecture to improve the scalability of the pure TDMA protocol while keeping the transmission delay low for large scale WSNs. The adoption of CDMA is inspired by recent research outcomes $[10,11]$ suggesting that the code-division multiplexing technology may be a suitable option for large scale WSNs because the use of properly designed codes will significantly reduce the channel access conflicts. The main idea of our hybrid TDMA/CDMA mechanism is to reduce the number of slots in a frame by allocating slots to active sensor groups rather than individual active sensors. The concurrent transmission of multiple active sensors inside a slot is resolved using CDMA technology. By proper power and rate control, the interference between nodes in the same slot can be minimized. To this end, we extend our optimization work in [28] by proposing two simplified power/time control (PTC) schemes. Compared with the optimal PTC algorithm in [28], the new schemes cut the number of control variables by almost $50 \%$ while still achieving above $90 \%$ of the energy efficiency provided by the old algorithm. Furthermore, in contrast to the centralized nature of the old algorithm, the simplified schemes can be implemented in a distributed way under moderate assistance of the $\mathrm{CH}$. Unlike the existing work on hybrid TDMA/CDMA for general wireless networks, which aims at maximizing system throughput $[25,16,17,21,18]$ or improving quality of service [19, 20, 22], here the target application scenario is WSNs and our efforts will focus on the energy efficiency aspect of this architecture.

For the proposed system architecture, we improve its energy efficiency in three different levels. First, in the network (or equivalently, the super-frame, as explained shortly) level, we eliminate the interference between clusters using spatial TDMA (STDMA) [23], where the interfering clusters in the system are assigned to different frames in a super-frame. Second, in the cluster (frame) level, we propose a group-based scheduling for the transmission of active nodes, i.e., we combine the active sensors into groups and assign slots to each group in such a way that the energy consumption of the frame is minimized. Finally, in the group (slot) level, we attempt to minimize the energy consumption of the slot by jointly optimizing the transmission powers and transmission durations (times) of the concurrent sensors in the group.

The main contributions of this paper are threefold. First, a vertex-coloring model is proposed to formulate the STDMA-based inter-cluster interference-control problem, whose solu- 
tion can be obtained efficiently using existing numerical algorithms in graph theory. Second, a heuristic algorithm was proposed to solve the optimal scheduling problem at the cluster level. The complexity of this algorithm is bounded by $O\left(N^{2}+N M(M-1)\right)$, where $N$ and $M$ are the number of active users in the cluster and the number of slots in the frame, respectively. By comparing with the discrete exhaustive search algorithm, we verified that this heuristic algorithm gives the near-optimal (or even optimal, as in most experiments) schedules with much less computing cost. Third, at the slot level, two simplified PTC algorithms are proposed based on our work in [28] to provide good energy efficiency at low control complexity.

The rest of this paper is organized as follows. The system model is described in Section 2. The optimization for the energy efficiency is presented in Section 3. The numerical examples and simulations are given in Section 4. Conclusions and discussions are given in Section 5.

\section{Model Description}

\subsection{System Architecture and Protocol Overview}

We consider a typical clustered WSN that conducts distributed sensing over some area [31, 29, 32], as shown in Figure 1. It consists of two types of nodes: A type-I node is a simple sensing node $(\mathrm{SN})$ that is responsible for sensing-related activities. Such nodes are small, low cost and disposable, and can be densely deployed across the sensing area. Neighboring SNs are organized into clusters using some clustering algorithm (e.g., see [24] and [25]). A type-II node, which has more energy conservation and stronger computing capability, is assigned to each cluster as a cluster head $(\mathrm{CH})$ and is responsible for receiving and processing sensing outcomes of SNs. We assume that each $\mathrm{CH}$ is within the communication range of all the SNs in its cluster. This assumption is usually supported by most existing clustering algorithms. For a SN, we assume that it communicates directly with the $\mathrm{CH}$ : It can transmit sensing data to or receive instructions from its $\mathrm{CH}$, but can not relay data from or instructions to a peer SN. Routing functions are supported by the CHs. A CH may collect data from the intra-cluster SNs, conduct signal processing (a.k.a., data fusion) on these raw data to create an application-specific view for the cluster, and then relay the fused data through intermediate CHs to the sink. Because SNs are usually deployed in large numbers, their relatively simple functionality helps lower the total cost of the network. On the other hand, because the number of CHs is much smaller than the number of SNs, it is quite feasible to replace $\mathrm{CHs}$ when their batteries are depleted. In 


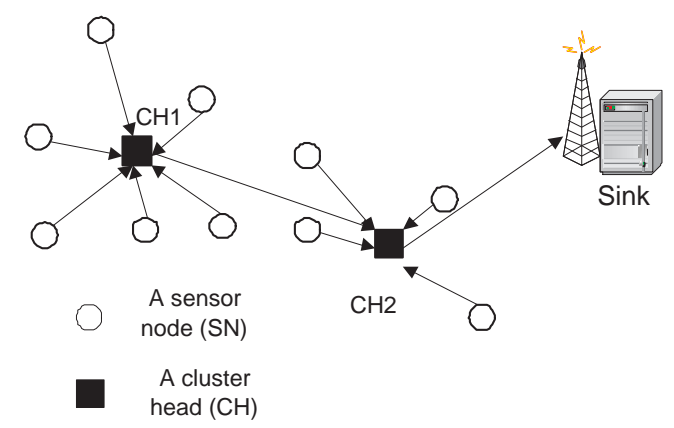

Figure 1: A topology example of the clustered WSN that contains two types of nodes.

addition, advanced battery-lifetime-prolonging technologies, such as solar-recharged batteries, can be applied to CHs without significantly increasing the total cost of the system. Such a hierarchical WSN has several applications, including localized key management [35], efficient querying [34], and landmark-based geographic routing [36], etc.

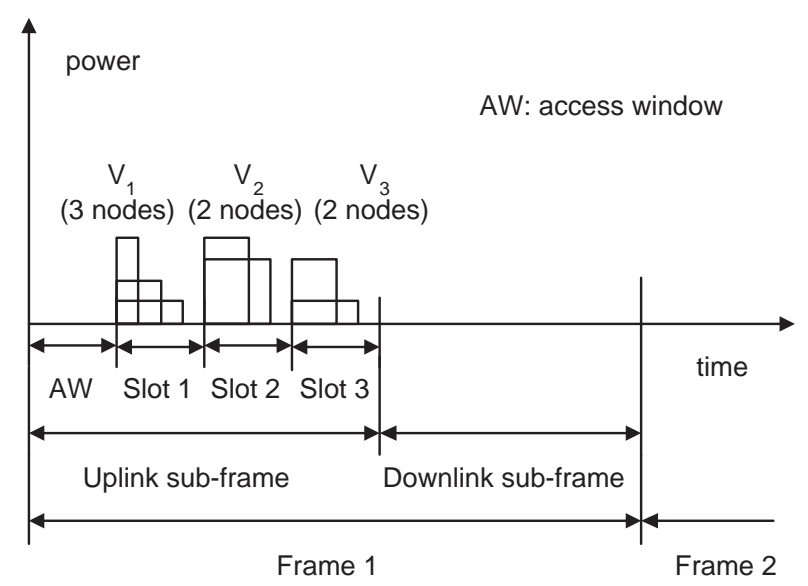

Figure 2: Illustration of the TDMA/CDMA setup $(N=7$ and $M=3)$.

The timing of the intra-cluster communication, i.e., the communication between SNs and their $\mathrm{CH}$, is based on a super-frame structure, as illustrated in Figure 2. The number of frames contained in each super-frame, i.e., $L$, is decided by a STDMA graph-coloring algorithm whenever the clustering or re-clustering is performed. In addition to $L$, this graph-coloring algorithm also decides an optimal cluster-to-frame assignment, by which any two interfering clusters are assigned to different frames in each super-frame. This treatment eliminates the inter-cluster interference, thus enables separate consideration for each cluster in its assigned frame. The graph-coloring algorithm will be elaborated in Section 3.1.

A frame consists of two sub-frames, namely, uplink sub-frame and downlink sub-frame, which are dedicated to the communications from the $\mathrm{SNs}$ to the $\mathrm{CH}$ and from the $\mathrm{CH}$ to the 
SNs, respectively. Taking the uplink sub-frame for an illustration (the case for downlink sub frame is similar), it consists of a channel access window followed by $M$ consecutive slots. At the beginning of the channel access window, the $\mathrm{CH}$ broadcasts a beacon message to synchronize its member SNs. A SN can also acquire the channel state information (CSI) of the link between itself and the $\mathrm{CH}$ by measuring the received signal strength of the beacon. Those active SNs that have data to send in the current frame will notify the $\mathrm{CH}$ about their transmission intention by sending a channel access request (CAR) packet to the $\mathrm{CH}$. A CAR packet contains such information as node id, number of data bits to be transmitted, and the CSI. Because the length of the CAR packet is extremely short, (e.g., a three-bit quantization of the CSI can give sufficient accuracy compared with the analog version of description [29]), these packets can be sent using p-persistent CSMA without causing significant collisions. Before the end of the access window, based on the information provided in the CAR packets, the CH executes a node scheduling algorithm to divide the active SNs into $M$ groups, each of which corresponds to a slot in the uplink sub-frame. For each group, CDMA is used to support the simultaneous transmission of SNs. The PTC parameters for each active SN, including the transmission powers and transmission times, are also computed by the $\mathrm{CH}$ when it schedules the nodes. Along with the transmission schedules, these PTC parameters are broadcasted by the CH to $\underline{\text { SNs before data transmission commences. A SN that receives its transmission information will }}$ turn on the radio and start transmission at the designated slot while sleeping in all other slots. Those SNs that have sent their CAR packets but do not receive their transmission information will realize that a collision happened on their CAR packets. They will turn off their radio, go into sleep, and retry the same process in the next super-frame. Because of the short packet length, the probability of collision between CAR packets is low, thus the number of SNs that have to refrain their transmission to the next super-frame is expected to be small. Note that by allowing such a retry, we implicitly assume that the underlying application can tolerate moderate delays, e.g., several super-frames.

To proceed with our analysis, we define the notations in Table 1. Because each cluster can be treated separately at the frame and slot levels (this point will be further explained in Section 3.1), these notations are defined over an arbitrary cluster. In addition, as is common for DS-CDMA systems, BPSK modulation is assumed. 
Table 1: Notations used in the analysis

\begin{tabular}{|l|l|}
\hline notation & definition \\
\hline$N$ & number of active SNs in the cluster \\
$M$ & number of slots in a frame \\
$\mathbf{V}_{i}$ & set of active SNs in the $i$ th group \\
$N_{i}$ & number of active SNs in the $i$ th group \\
node $(i, j)$ & the $i$ th active SN in the $j$ th group \\
$B_{i j}$ & number of bits to be transmitted from node $(i, j)$ in the underlying frame \\
$P_{t i j}$ & transmission power of node $(i, j)$ in transmitting $B_{i j}$ bits \\
$T_{i j}$ & transmission duration for node $(i, j)$ in transmitting $B_{i j}$ bits \\
$\gamma_{i j}$ & lower bound on the received bit-energy-to-interference ratio for node $(i, j)$ \\
$E_{i j}$ & energy consumption at node $(i, j)$ for transmitting $B_{i j}$ bits \\
$P_{c i j}$ & power of the circuit at node $(i, j)$ when the circuit is on \\
$P_{\max }$ & upper bound on the transmission power $P_{t i j}$ \\
$T_{s}$ & slot length \\
$W$ & spread-spectrum bandwidth of CDMA $(\mathrm{Hz})$ \\
$N_{0}$ & single-sided power spectrum density of $\mathrm{AWGN} \mathrm{(Watt/Hz)}$ \\
\hline
\end{tabular}

\subsection{Energy Consumption Model}

We use a comprehensive model to describe the energy consumption of the communications in the system. Consider node $(i, j)$ in an arbitrary cluster. For each data transmission, energy consumption at this node $\left(E_{i j}\right)$ consists of a transmission component and a circuit component, i.e.,

$$
E_{i j}=\left(P_{t i j}+P_{c i j}\right) T_{i j}
$$

where $P_{c i j}$ is the power consumed by the circuit at sensor $(i, j)$. Unlike the communications in conventional cellular or mobile ad hoc networks, where the communication range is long, it is usually short-range communication in WSNs due to the high density of the nodes. In this environment, the circuit energy consumption is comparable with the transmission energy consumption, thus should not be ignored in the energy optimization. Following a similar model to the one in [26], $P_{c i j}$ can be written as

$$
P_{c i j}=\alpha_{i j}+\left(\frac{1}{\eta}-1\right) P_{t i j}
$$

where $\alpha_{i j}$ is a transmit-power-independent component that accounts for the power consumed by the digital-to-analog converter, the signal filters, and the modulator; and $\eta$ is the efficiency factor of the power amplifier, which represents the fraction of power that the amplifier can use to transmit the signal. 
Substituting (2) into (1), the energy consumption of node $(i, j)$ is given by

$$
E_{i j}=\frac{1}{\eta} P_{t i j} T_{i j}+\alpha_{i j} T_{i j}=\frac{1}{\eta}\left(P_{t i j}+\alpha_{c i r i j}\right) T_{i j}
$$

where $\alpha_{c i r i j}=\eta \alpha_{i j}$ is the equivalent circuit power consumption. For $N$ active SNs in the cluster, the total energy consumption of the cluster in the underlying frame is

$$
E_{\text {total }}=\sum_{j=1}^{M} \sum_{i=1}^{N_{j}} E_{i j}=\frac{1}{\eta} \sum_{j=1}^{M} \sum_{i=1}^{N_{j}}\left(P_{t i j}+\alpha_{c i r i j}\right) T_{i j} .
$$

The problem of joint optimal PTC and scheduling in a cluster is to minimize $E_{\text {total }}$ by controlling the scheduling variables $\mathbf{V}_{j}$ 's and the transmission variables $P_{t i j}$ 's and $T_{i j}$ 's, $i=$ $1, \ldots, N_{j}$ and $j=1, \ldots, M$. The scheduling algorithm and the optimization over transmission powers and times will be elaborated in Sections 3.2 and 3.3, respectively.

\section{Energy Efficiency Improvement for Hybrid TDMA/CDMA Transmission}

In this section, we consider the problem of improving the energy efficiency of the proposed system on transmitting a given amount of data in a super-frame. Ultimately, the energy consumption comes from the sum of the transmission and circuit energy consumptions in each slot of the super-frame, where simultaneous transmissions of active SNs are based on CDMA. Due to the multiple access interference (MAI) of CDMA, deciding the optimal transmission powers and times for the concurrent SNs is not straightforward. For example: increasing the transmission power of one node is beneficial to increasing its transmission rate, thus reducing the transmission time, leading to lower circuit energy consumption of that node; however, all other concurrent nodes have to increase their transmission power, and accordingly, the transmission energy, to combat the added MAI. Thus the increased transmission energy consumption may cancel out the energy savings from the circuits. On the other hand, deciding the optimal scheduling of nodes is NP-hard. It is well known that the exhaustive search algorithm, which explores all the possible combinations of the concurrent nodes, has an exponential complexity with the numbers of slots and nodes.

The methodology employed in this section in tackling this NP-hard problem is to decompose the original problem into three sequential sub problems, each of which has a smaller variable space than the original problem and can be approximately solved by efficient algorithms. Specifically, the energy improvement is conducted sequentially at three different levels: Firstly, the 
inter-cluster interference is eliminated through STDMA at the network level; this treatment enables us to reduce the energy consumption of each cluster separately; Secondly, for a cluster, the intra-cluster node scheduling is decided using iterative method; Finally, for a slot, the PTC for concurrent nodes is decided based on some optimization formulations.

\subsection{Vertex-coloring for Clusters}

We handle the inter-cluster interference-control problem in this section using Spatial TDMA [23]. STDMA is a generalization of the TDMA protocol, where the transmission cycles are organized into super-frames. A super-frame consists of several frames, each of which is allocated to a set of non-interfering clusters in the network.

The optimal STDMA of clusters can be modeled as a vertex-coloring problem [27]. Consider a cluster-interfering graph $\mathbf{G}=(\mathbf{C}, \mathbf{E})$, where $\mathbf{C}$ represents the set of clusters in the network and $\mathbf{E}$ is a diagonal and symmetric matrix of size $|\mathbf{C}|$, whose elements represent the interfering relation between any two clusters in $\mathbf{C}$. For an element $e_{i, j} \in \mathbf{E}, e_{i, j}=1$ if the $\mathrm{CH}$ in cluster $j$ can receive the signal from any $\mathrm{SN}$ in cluster $i$ or the $\mathrm{CH}$ in cluster $i$ can receive the signal from any $\mathrm{SN}$ in cluster $i$. Otherwise $e_{i, j}=0$. The optimization problem is to find the minimum number of frames, $L$, such that every cluster is assigned with a frame and any two clusters whose $e_{i, j}$ equals 1 are assigned with different frames. This is exactly the well-known vertexcoloring problem in the graph theory. Particularly, it has been shown in [27] that in reality the interfering clusters can be well modeled as a planar graph, whereby a cluster only interferes with its adjacent clusters. In this situation, the famous four-color theorem states that four frames are enough to separate those interfering clusters. Accordingly, polynomial-time algorithms were given in [27] for the optimal frame assignment problem.

A possible executer of the vertex-coloring algorithm is the sink of the system. After the formation of clusters or the execution of re-clustering, each $\mathrm{CH}$ reports its interference condition, i.e., whether it receives signal from the SNs that do not belong to its cluster, to the sink. Based on this interference report, the sink generates the cluster-interfering graph $\mathbf{G}$ and apply vertexcoloring algorithm to $\mathbf{G}$. The results are used to set up the super-frame and are notified to every $\mathrm{CHs}$ in the system. 


\subsection{Energy-efficient Node Scheduling}

The graph coloring of clusters introduced in the previous section enables us to study the energy consumption of each cluster separately. For an arbitrary cluster, the main goal of the node scheduling process is to decide the grouping of active SNs in such a way that the total energy consumption in the current frame is minimized. Since different PTC policies can be applied to the same node combinations, leading to different energy consumptions, the optimal node scheduling is intrinsically related to the PTC algorithm employed in each slot. Obviously, for a pre-defined PTC policy, the optimal node scheduling can be found by alternately executing the following two procedures: First, for a given node schedule, find the optimal transmission powers and times under the pre-defined PTC policy that provides the conditional (conditioned on the given node combination) minimum energy consumption in each slot. Second, change the combination of nodes and execute the first procedure over the updated schedule. The node combination that provides the minimum energy consumption among all possible combinations should be picked as the optimal schedule. Formally, this node scheduling problem is presented as follows.

For a given node schedule $\left(\mathbf{V}_{1}, \ldots, \mathbf{V}_{M}\right)$, if it is feasible (the definition of feasibility is given shortly later), we apply the PTC algorithm described in Sections 3.3 to every slot $\mathbf{V}_{i}$, $i=1, \ldots, M$, to minimize the energy consumption of the frame:

$$
E_{\text {total }}^{\text {PTColicy }}\left(\mathbf{V}_{1}, \ldots, \mathbf{V}_{M}\right) \stackrel{\text { def }}{=} \sum_{i=1}^{M} E_{i}^{\text {PTCpolicy }}\left(\mathbf{V}_{i}\right)
$$

where $E_{i}^{P T C p o l i c y}\left(\mathbf{V}_{i}\right)$ is a function of the concurrent nodes in group $\mathbf{V}_{i}$ (which are transmitted in the $i$ th slot) and denotes the minimum energy consumption for this group under the PTC policy employed for the transmission. Here, we define that a node group $\mathbf{V}_{i}$ is feasible for a PTC policy iff the power allocation of every member node has a positive value under the employed PTC algorithm. Furthermore, we define that a node schedule is feasible for a PTC policy iff every node group in this schedule is feasible under the PTC policy. The feasibility conditions, along with the specific forms of the function $E_{i}^{P T C p o l i c y}\left(\mathbf{V}_{i}\right)$ under various PTC algorithms, will be given in Section 3.3 when we present the PTC algorithms.

Mathematically, the problem of finding the optimal node schedule that minimizes $E_{\text {total }}^{\text {policy }}\left(\mathbf{V}_{1}, \ldots, \mathbf{V}_{M}\right)$ 
in $(5)$ is formulated as

$$
\left\{\begin{array}{l}
\operatorname{minimize}_{\left\{\mathbf{V}_{1}, \ldots, \mathbf{V}_{M}\right\}} E_{\text {total }}^{P T C p o l i c y}\left(\mathbf{V}_{1}, \ldots, \mathbf{V}_{M}\right) \\
\text { s.t. } \\
\mathbf{V}_{i} \text { is feasible for the PTC policy }, i=1, \ldots, M \\
\mathbf{V}_{i} \cap \mathbf{V}_{j}=\emptyset, i \neq j \\
\sum_{i=1}^{M}\left|\mathbf{V}_{i}\right|=N .
\end{array}\right.
$$

The discrete nature of the control variables in (6) naturally lends this problem to the discrete exhaustive search (DES) algorithm, which explores all possible node combinations to solve for the optimal schedule. However, it is easy to show that the total number of all possible node combinations is in the order of $O\left(2^{N(M-1)}\right)$, thus this algorithm has a double exponential complexity. With the growth of the network size in both $N$ and $M$, such prohibitive complexity deprives the applicability from the DES algorithm for any practical usage.

We now propose a heuristic algorithm, which has polynomial time complexity and can approximately solve the optimization problem. An iterative method is taken to alter node combinations in such a way that the frame energy consumption keeps decreasing with each iteration. More specifically, in each iteration, the schedule is changed by shifting one node from its original group to a new group, or swapping two nodes between two groups, provided that this change will result in a reduced frame energy consumption. Initially, suppose we have an arbitrary feasible node schedule. We first conduct node shifting in each iteration. Set $\mathbf{V}_{1}$ as the source group and $\mathbf{V}_{2}$ as the destination group. For every node in the source group, we calculate the energy consumption variations when it is shifted to the destination group. If this altering leads to feasible groups and reduced total energy consumption, we will keep this schedule change. If not, we just let this node stay at the source group. After we complete this node shifting from $\mathbf{V}_{1}$ to $\mathbf{V}_{2}$, we change the destination to $\mathbf{V}_{3}$, and repeat the same procedure. And so on. We consider such node shifting from every group to every other group by changing the source and destination groups sequentially and respectively, until all possible source-destination combinations are visited. It is easy to show that the number of all possible shifting is bounded by $N M(M-1)$. Note that the schedule reached after this node shifting (NSF) phase is related to the initial schedule settings, so it is not necessarily unique. This is further refined by performing the node-swapping (NSW) phase: we consider swapping the group assignment of all pairs of nodes that belong to different groups if this swapping results in feasible groups and a reduction in the total energy consumption. For each node in $\mathbf{V}_{1}$, the number of pairs to be considered for swapping is $N-N_{1}$, for a total of $N_{1}\left(N-N_{1}\right)$ for all the 
nodes in $\mathbf{V}_{1}$. Similarly, for $\mathbf{V}_{2}$, there are $N_{2}\left(N-N_{2}\right)$ pairs to consider. A simple combinatorial argument shows that the total number of pairs to consider is bounded by $N^{2}$. Combining NSF and NSW phases, the total complexity of the suggested greedy algorithm is bounded by $O\left(N^{2}+N M(M-1)\right)$. A pseudo-code description of this greedy algorithm is given in Table 2. The accuracy of this heuristic algorithm will be evaluated later in the numerical examples.

\subsection{Joint PTC for Concurrent SNs}

Given a node schedule $\left(\mathbf{V}_{1}, \ldots, \mathbf{V}_{M}\right)$, we decide the transmission powers and times for the concurrent $\mathrm{SNs}$ in each slot to conditionally minimize the energy consumption of the slot. The results will be plugged into the iterative algorithm in Section 3.2 to decide the energy consumption of each node combination.

Without loss of generality, we focus on one group, say $\mathbf{V}_{1}$, and drop the group dependence from our notations. Suppose that there are $N$ nodes in this group. Let $B_{i}$ be the number of bits that node $i$ needs to transmit in the underlying slot. The primary objective of our work is to find the optimal transmission power $P_{t i}^{o}$ and transmission time $T_{i}^{o}$ for each node $i$ such that the total energy consumed in transmitting $\sum_{i=1}^{N} B_{i}$ bits is minimized while the QoS requirement (in terms of the quality of the received signal) of each transmission is satisfied. We consider three joint power/time control schemes that provide different degrees of freedom in controlling power and time.

1. PTC-IPT Scheme: The PTC with independent power and times (PTC-IPT) scheme [28] provides complete freedom in controlling the transmission power and time of every node. For a group of $N$ nodes, there are $2 N$ independent control variables, i.e., $P_{t i}$ and $T_{i}$, $i=1, \ldots, N$, which need to be optimized to minimize the total energy consumption. More specifically, the optimization problem is formulated as

$$
\left\{\begin{array}{l}
\operatorname{minimize}_{\left\{\mathbf{P}_{t}, \mathbf{T}\right\}} \sum_{i=1}^{N}\left(P_{t i}+\alpha_{c i r i}\right) T_{i} \\
\text { s.t. } \\
\left(\frac{E_{b}}{I_{0}}\right)_{i} \geq \gamma_{i}, \quad i=1, \ldots, N \\
0 \leq T_{i} \leq T_{S}, \quad i=1, \ldots, N \\
0 \leq P_{t i} \leq P_{\max }, \quad i=1, \ldots, N
\end{array}\right.
$$

where $\mathbf{P}_{t} \stackrel{\text { def }}{=}\left(P_{t 1}, \ldots, P_{t N}\right)$ is the transmit power vector, $\mathbf{T} \stackrel{\text { def }}{=}\left(T_{1}, \ldots, T_{N}\right)$ is the trans-

mission time vector, and $\left(\frac{E_{b}}{I_{0}}\right)_{i}$ is the received bit-energy-to-interference-density-ratio at 
Input: an arbitrary feasible schedule $\left(\mathbf{V}_{1}, \ldots, \mathbf{V}_{M}\right)$

NSF: $\quad$ for source $=1$ to $M$

for $\forall i \in \mathbf{V}_{\text {source }}$

energy_variation $=0$, shift_destination $=0$

for destination $=1$ to $M$

if source $\neq$ destination

$\mathbf{V}_{\text {source }}^{\prime}=\mathbf{V}_{\text {source }}-\{i\}$

$\mathbf{V}_{\text {destination }}^{\prime}=\mathbf{V}_{\text {destination }}+\{i\}$

current_reduction $=\operatorname{energy}\left(\mathbf{V}_{\text {source }}^{\prime}\right)+\operatorname{energy}\left(\mathbf{V}_{\text {destination }}^{\prime}\right)$

$-\operatorname{energy}\left(\mathbf{V}_{\text {source }}\right)-\operatorname{energy}\left(\mathbf{V}_{\text {destination }}\right)$

if current_reduction < energy_variation

energy_variation $=$ current_reduction

shift_destination $=$ destination

endif; endif; endfor

if energy_variation $<0$

$$
\begin{aligned}
& \mathbf{V}_{\text {source }}=\mathbf{V}_{\text {source }}-\{i\} \\
& \mathbf{V}_{\text {shift_destination }}=\mathbf{V}_{\text {shift_destination }}+\{i\}
\end{aligned}
$$

endif; endfor; endfor

NSW: for source $=1$ to $M$

for $\forall i \in \mathbf{V}_{\text {source }}$

energy_variation $=0$, swap_group $=0$, swap_node $=0$

for destination $=1$ to $M$

if source $\neq$ destination

for $\forall j \in \mathbf{V}_{\text {destination }}$

$\mathbf{V}_{\text {source }}^{\prime}=\mathbf{V}_{\text {source }}-\{i\}+\{j\}$

$\mathbf{V}_{\text {destination }}^{\prime}=\mathbf{V}_{\text {destination }}-\{j\}+\{i\}$

if $\mathbf{V}_{\text {source }}^{\prime}$ and $\mathbf{V}_{\text {destination }}^{\prime}$ are feasible

current_reduction $=\operatorname{energy}\left(\mathbf{V}_{\text {source }}^{\prime}\right)+\operatorname{energy}\left(\mathbf{V}_{\text {destination }}^{\prime}\right)$

$-\operatorname{energy}\left(\mathbf{V}_{\text {source }}\right)-\operatorname{energy}\left(\mathbf{V}_{\text {destination }}\right)$

if current_reduction < energy_variation

energy_reduction $=$ current_reduction

swap_group $=$ destination, swap_node $=j$

endif; endif; endfor; endif; endfor

if energy_variation $<0$

$$
\mathbf{V}_{\text {source }}=\mathbf{V}_{\text {source }}-\{i\}+\{\text { swap_node of swap_group }\}
$$

$\mathbf{V}_{\text {swap_group }}=\mathbf{V}_{\text {swap_group }}-\{$ swap_node $\}+\{i\}$

endif; endfor; endfor

Output: $\left(\mathbf{V}_{1}, \ldots, \mathbf{V}_{M}\right)$

Table 2: Pseudo-code for computing the optimal solution for the transmit powers and times. 
node $o$ for sensor $i$ :

$$
\begin{aligned}
\left(\frac{E_{b}}{I_{0}}\right)_{i} & =\frac{W}{R_{i}} \frac{h_{i} P_{t i}}{\delta \sum_{j=1, j \neq i}^{N} h_{j} P_{t j}+N_{0} W} \\
& =\frac{W}{B_{i}} \frac{h_{i} P_{t i} T_{i}}{\delta \sum_{j=1, j \neq i}^{N} h_{j} P_{t j}+N_{0} W}
\end{aligned}
$$

where $R_{i}=\frac{B_{i}}{T_{i}}$ is the transmission rate under the assumption of BPSK modulation, $h_{i}$ is the channel gain, and $\delta$ is the orthogonality factor, representing multiple access interference (MAI) from the imperfectly orthogonal spreading codes and the asynchronous chips across simultaneous transmitting nodes. Typical values for $\delta$ are $\frac{2}{3}$ and 1 for a chip of rectangular and sinoide shapes, respectively.

2. PTC-UT Scheme: Under PTC with unified transmission time (PTC-UT) scheme, node $o$ specifies the $N$ transmission powers and a common transmission time for all nodes. So there are $N+1$ independent variables, i.e., $P_{t i}$ for $i=1, \ldots, N$ and $T_{1}=T_{2}=$ $\ldots=T_{N}$, which need to be optimized to minimize the total energy consumption. As will become clearer later on, under PTC-UT, the optimal transmit power at each node can be computed locally based on some common parameters broadcasted by node $o$. The distributed nature of this scheme reduces the control overhead and simplifies system design. For the PTC-UT scheme, the optimization problem can be expressed as in (7) with the additional constraint:

$$
T_{1}=T_{2}=\ldots=T_{N}
$$

3. PTC-USG Scheme: For PTC with unified spreading gain (PTC-USG) scheme, node $o$ specifies the $N$ transmission powers and the $N$ transmission durations for all nodes in such a way that all the $N$ transmissions have the same data rate. Accordingly, there are $N+1$ independent control variables, i.e., $P_{t i}$ for $i=1, \ldots, N$ and $R \stackrel{\text { def }}{=} \frac{B_{1}}{T_{1}}=\frac{B_{2}}{T_{2}}=\ldots=\frac{B_{N}}{T_{N}}$, which need to be optimized. Similar to PTC-UT, PTC-USG can be implemented in a distributed fashion. By taking advantage of the common spreading gain $\left(\frac{W}{R}\right)$ across different nodes, the implementation can be further simplified by assigning in each cycle the same family of spreading codes for all the sensors. For the PTC-USG scheme, the optimization has the same form as (7) with the additional constraint:

$$
\frac{B_{1}}{T_{1}}=\frac{B_{2}}{T_{2}}=\ldots=\frac{B_{N}}{T_{N}} .
$$


Although at the first glance, PTC-UT and PTC-USG may be seen as two straightforward simplifications of PTC-IPT, by studying the specific structure of their analytical results, we will reveal their non-straightforward features: They reserve most of (above 90\% in our numerical examples) the energy efficiency provided by PTC-IPT while significantly reducing the implementation complexities, e.g., cutting almost half of the control variables and being capable of distributed implementation. Such features indicate the insensitivity of the energy efficiency to the transmission time control (or equivalently, the transmission rate control) of individual nodes when the transmission power control has been performed. Such an observation reconfirms and enhances the conclusion made in [30], where it has been found that for the single-link case, rate control can only contribute marginal improvement to the link efficiency when power control has been conducted. The significance of our work is that it extends the validity of this conclusion to multiple access systems and to the scenario that not only the communication energy, but also the circuit energy consumption are accounted for in the performance metrics.

The analysis for these schemes is elaborated as follows. Because of the cross-product of $\mathbf{P}_{t}$ and $\mathbf{T}$ in the objective function of (7), none of the optimization under the three PTC algorithms is convex. However, with some algebraic manipulations, it has been shown in [28] that the objective function and the constraints in (7) can be put in the forms of posynomials in $\mathbf{P}_{t}$ and $\mathbf{T}$, so that the resulting optimization problem is a standard geometric program (GP) [33]. Efficient numerical algorithms for solving GPs, e.g., interior point algorithm, are readily available.

Rather than relying on a numerical approach, we concentrate on an analytical solution to this problem, with the hope that it may be implementable for real-time control in practical applications. The analytical solution is obtained by decoupling the joint power/time optimization problem into two sequential sub-problems. The first sub-problem is a parametric linear optimization on the transmission power with the transmission time $\mathbf{T}$ being the parameter. Then, the optimization on $\mathbf{T}$ is approximately formulated as a convex problem, whose solution is derived either through sequential algorithm (for PTC-IPT) or in closed form (for PTC-UT and PTC-USG). Mathematically, this decoupling process is presented as follows.

\subsubsection{Sub-Problem 1: Parametric Solution of Optimal Transmission Powers}

Because the formulations for PTC-UT and PTC-USG can be derived from that of PTC-IPT with an additional constraint on $\mathbf{T}$, we first consider the variable-decoupling of (7). 
Treating the transmission time vector $\mathbf{T}$ as a given system parameter with $T_{i} \leq T_{S}$, (7) is equivalent to the following linear programming problem:

$$
\left\{\begin{array}{l}
\operatorname{minimize}_{\left\{P_{t 1}, \ldots, P_{t N}\right\}} \sum_{i=1}^{N} P_{t i} T_{i} \\
\text { s.t. } \\
\left(1+\frac{\delta B_{i} \gamma_{i}}{W T_{i}}\right) h_{i} P_{t i}-\frac{\delta B_{i} \gamma_{i}}{W T_{i}} \sum_{j=1}^{N} h_{j} P_{t j} \geq \frac{B_{i} \gamma_{i} N_{0}}{T_{i}} \\
P_{t i} \leq P_{\max }, \quad i=1, \ldots, N .
\end{array}\right.
$$

In [28], we have derived the parametric optimal solution to (11) in terms of the transmission time $\mathbf{T}$

$$
P_{t i}=\frac{\delta^{-1} h_{i}^{-1} g_{i}}{1-g_{\Sigma}}, \quad i=1, \ldots, N
$$

where $P_{t i}$ has been normalized with respect to the energy of background AWGN, $g_{\Sigma} \stackrel{\text { def }}{=} \sum_{i=1}^{N} g_{i}$, and $g_{i}$ is the power index of node $i$ :

$$
g_{i} \stackrel{\text { def }}{=} \frac{\delta B_{i} \gamma_{i}}{W T_{i}+\delta B_{i} \gamma_{i}}
$$

Accounting for the maximum transmit power constraint, the necessary conditions for the existence of the optimal solution are given by [28]:

$$
g_{i} \leq \delta h_{i} P_{\max }, \quad i=1, \ldots, N
$$

and

$$
g_{\Sigma} \leq \frac{\delta P_{\max } h_{\Sigma}}{1+\delta P_{\max } h_{\Sigma}}<1
$$

where $h_{\Sigma} \stackrel{\text { def }}{=} \sum_{i=1}^{N} h_{i}$.

Because we have not specified any additional constraints on $T_{i}$ 's in our efforts above, the parametric treatment of (11), the result in (12), and the constraints in (14) and (15) also apply to the formulations for PTC-UT and PTC-USG.

\subsubsection{Sub-Problem 2: Optimization of Transmission Times}

From (13), it is clear that for given system parameters $B_{i}, \gamma_{i}, W$, and $\delta$, the power index $g_{i}$ and the transmission time $T_{i}$ are equivalent measures in the sense that there is a one-to-one mapping between $g_{i}$ and $T_{i}$ :

$$
T_{i}=\frac{\delta B_{i} \gamma_{i}}{W g_{i}}\left(1-g_{i}\right)
$$

In the following optimization, it is more mathematically convenient to work with $g_{i}$. Let $\mathbf{g} \stackrel{\text { def }}{=}\left(g_{1}, \ldots, g_{N}\right)$. The problem of determining the optimal value of $\mathbf{g}$ is now considered. 


\subsubsection{PTC-IPT Scheme}

In [28], we have shown that the problem of determining the optimal value of $\mathbf{g}$ can be approximately formulated as the following convex problem

$$
\left\{\begin{array}{l}
\text { minimize }_{\{\mathbf{g}\}} \frac{K}{1-g_{\Sigma}}+\sum_{i=1}^{N} \frac{\alpha_{c i r i} A_{i}}{g_{i}}-\sum_{i=1}^{N} \alpha_{c i r i} A_{i} \\
\text { s.t. } \\
\frac{\delta B_{i} \gamma_{i}}{\delta B_{i} \gamma_{i}+W T_{i}^{l i m i t}} \leq g_{i} \leq \delta h_{i} P_{\max }, \quad i=1, \ldots, N \\
\sum_{i=1}^{n} g_{i} \leq \frac{\delta P_{\max } h_{\Sigma}}{1+\delta P_{\max } h_{\Sigma}},
\end{array}\right.
$$

where $A_{i} \stackrel{\text { def }}{=} \frac{\delta B_{i} \gamma_{i}}{W}$ is a node-dependent constant and $K \stackrel{\text { def }}{=} \sum_{i=1}^{N} \delta^{-1} h_{i}^{-1} A_{i}$ is a group-dependent constant.

We have proved in [28] that the optimal solution to (17) can be derived by first solving the un-bounded optimization problem where the upper and lower bounds on $g_{i}$ is not imposed, and then iteratively fixing those variables that exceed their bounds. Particular, when the traffic load is reasonably smaller than the network's capacity, the optimal transmission time is located within the polyhedron depicted by the constraints of (17). In this case, the optimal solution is given by

$$
g_{i}^{o}=\frac{\sqrt{\alpha_{c i r i} A_{i}}}{\sqrt{K}+\sum_{i=1}^{N} \sqrt{\alpha_{\text {ciri }} A_{i}}}, \quad i=1, \ldots, N .
$$

Having determined $g_{i}^{o}$, the optimal transmit power and transmission time are derived by substituting (18) into (12) and (16), respectively

$$
\begin{aligned}
P_{t i}^{o(P T C-I P T)} & =\frac{\delta^{-1} h_{i}^{-1} g_{i}^{o}}{1-g_{\Sigma}^{o}}, \\
T_{i}^{o(P T C-I P T)} & =\frac{\delta B_{i} \gamma_{i}}{W g_{i}^{o}}\left(1-g_{i}^{o}\right), \quad i=1, \ldots, N,
\end{aligned}
$$

where $g_{\Sigma}^{o} \stackrel{\text { def }}{=} \sum_{i=1}^{N} g_{i}^{o}$.

\subsubsection{PTC-UT Scheme}

A typical WSN application is usually characterized by low data rate and low energy consumption at each SN. According to (13), this condition implies $g_{i} \ll 1$ for a node $i$. Therefore, according to (9), we have

$$
\begin{gathered}
T_{i} \approx \frac{\delta B_{1} \gamma_{1}}{W g_{1}} \\
g_{i}=\frac{B_{i} \gamma_{i}}{B_{1} \gamma_{1}} g_{1}, \quad i=1, \ldots, N .
\end{gathered}
$$


Substituting (21), (22), (12) and the constraints (14) and (15) into (7), the problem of determining the optimal transmission time under PTC-UT can be formulated by

$$
\left\{\begin{array}{l}
\text { minimize }_{\left\{g_{1}\right\}} f\left(g_{1}\right) \stackrel{\text { def }}{=} \frac{C}{1-D g_{1}}+\frac{E}{g_{1}} \\
\text { s.t. } \\
g_{1}^{\text {low }} \leq g_{1} \leq g_{1}^{\text {upp }} .
\end{array}\right.
$$

where $C \stackrel{\text { def }}{=} \frac{1}{W} \sum_{i=1}^{N} h_{i}^{-1} B_{i} \gamma_{i}, D \stackrel{\text { def }}{=} \frac{\sum_{i=1}^{N} B_{i} \gamma_{i}}{B_{1} \gamma_{1}}, E \stackrel{\text { def }}{=} \frac{\delta B_{1} \gamma_{1} \sum_{i=1}^{N} \alpha_{c i r i}}{W}, g_{1}^{\text {low }} \stackrel{\text { def }}{=} \max _{i}\left\{\frac{\delta B_{1} \gamma_{1}}{\delta B_{i} \gamma_{i}+W T_{i}^{\text {limit }}}\right\}$, and $g_{1}^{\text {upp }} \stackrel{\text { def }}{=} \min \left\{\frac{\delta P_{\max } h_{\Sigma} B_{1} \gamma_{1}}{\left(1+\delta P_{\max } h_{\Sigma}\right) \sum_{j=1}^{N} B_{j} \gamma_{j}}, \min _{i}\left\{\frac{\delta h_{i} P_{\max } B_{1} \gamma_{1}}{B_{i} \gamma_{i}}\right\}\right\}$ are system-defined constants. Note that once the optimal $g_{1}$ is found, the optimal $g_{i}, i=2, \ldots, N$, can be computed from (22).

By taking the second-order derivation of $f\left(g_{1}\right)$ in $(23)$, we can prove the function $f\left(g_{1}\right)$ is strictly convex and must have only one unconstrained minimum solution, which is given by solving the following equation for $g_{1}$

$$
f^{\prime}\left(g_{1}\right)=\frac{C D}{\left(1-g_{1} D\right)^{2}}-\frac{E}{g_{1}^{2}}=0 .
$$

Solving (24), the unconstrained minimum solution is given by

$$
g_{u 1}^{o}=\frac{\sqrt{E}}{\sqrt{C D}+\sqrt{E} D}
$$

Accounting for the upper and lower bounds given in (23), the constrained optimal solution to $(23)$ is given by

$$
g_{1}^{o}= \begin{cases}g_{u 1}^{o}, & g_{1}^{\text {low }} \leq g_{u 1}^{o} \leq g_{1}^{u p p} \\ g_{1}^{\text {low }}, & g_{u 1}^{o}<g_{1}^{\text {low }} \\ g_{1}^{u p p}, & g_{u 1}^{o}>g_{1}^{u p p}\end{cases}
$$

Having determined $g_{1}^{o}$, the optimal transmission times and powers are given by substituting $g_{1}^{o}$ into (21) and (12), resulting in

$$
\begin{aligned}
T_{i}^{o(P T C-U T)} & =\frac{\delta B_{1} \gamma_{1}}{W g_{1}^{o}}, \quad i=1, \ldots, N \\
P_{t i}^{o(P T C-U T)} & =\frac{\delta^{-1} h_{i}^{-1} B_{i} \gamma_{i} g_{1}^{o}}{B_{1} \gamma_{1}-g_{1}^{o} \sum_{j=1}^{N} B_{j} \gamma_{j}} .
\end{aligned}
$$

\subsubsection{PTC-USG Scheme}

Denote $R \stackrel{\text { def }}{=} \frac{B_{1}}{T_{1}}=\frac{B_{2}}{T_{2}}=\ldots=\frac{B_{N}}{T_{N}}$. The value of $g_{i}$ can be approximately presented in terms of $R$ as

$$
g_{i} \approx \frac{\delta \gamma_{i} R}{W}, \quad i=1, \ldots, N
$$


Substituting $T_{i}=\frac{B_{i}}{R},(29)$, (12) and the constraints (14) and (15) into (7), the problem of determining the optimal $R$ under PTC-USG scheme is formulated as

$$
\left\{\begin{array}{l}
\operatorname{minimize}_{\{R\}} l(R) \stackrel{\text { def }}{=} \frac{F}{1-R G}+\frac{H}{R} \\
\text { s.t. } \\
R^{\text {low }} \leq R \leq R^{\text {upp }} .
\end{array}\right.
$$

where $F \stackrel{\text { def }}{=} \frac{\sum_{i=1}^{N} h_{i}^{-1} \gamma_{i} B_{i}}{W}, G \stackrel{\text { def }}{=} \frac{\delta \gamma_{\Sigma}}{W}, H \stackrel{\text { def }}{=} \sum_{i=1}^{N} \alpha_{\text {ciri }} B_{i}, \gamma_{\Sigma} \stackrel{\text { def }}{=} \sum_{i=1}^{N} \gamma_{i}, R^{\text {low }} \stackrel{\text { def }}{=} \max _{i}\left\{\frac{W B_{i}}{\delta B_{i} \gamma_{i}+W T_{i}^{\text {limit }}}\right\}$, and $R^{\text {upp }} \stackrel{\text { def }}{=} \min \left\{\frac{P_{\max } h_{\Sigma} W}{\left(1+\delta P_{\max } h_{\Sigma}\right) \gamma_{\Sigma}}, \min _{i}\left\{\frac{W h_{i} P_{\max }}{\gamma_{i}}\right\}\right\}$ are system-defined constants.

An observation of the objective function $l(R)$ in $(30)$ shows that it has the same form as $f\left(g_{1}\right)$ in $(23)$. Therefore, $l(R)$ must be strictly convex and has only one unconstrained optimal solution

$$
R_{u}^{o}=\frac{\sqrt{H}}{\sqrt{F G}+\sqrt{H} G} .
$$

Accounting for the upper and lower bounds in (30), the constrained optimal solution to (30) is given by

$$
R^{o}=\left\{\begin{array}{l}
R_{u}^{o}, \quad R^{\text {low }} \leq R_{u}^{o} \leq R^{\text {upp }} \\
R^{\text {low }}, \quad R_{u}^{o}<R^{\text {low }} \\
R^{\text {upp }}, \quad R_{u}^{o}>R^{\text {upp }}
\end{array}\right.
$$

Substituting $R^{o}$ and (29) into (12), the optimal transmission power and transmission time under PTC-USG scheme are given by

$$
\begin{aligned}
T_{i}^{o(P T C-U S G)} & =\frac{B_{i}}{R^{o}}, \quad i=1, \ldots, N, \\
P_{t i}^{o(P T C-U S G)} & =\frac{h_{i}^{-1} \gamma_{i} R^{o}}{W-R^{o} \delta \gamma_{\Sigma}}, \quad i=1, \ldots, N .
\end{aligned}
$$

\subsubsection{Energy Efficiency Comparison}

Based on the expressions of the optimal transmission powers and times derived in previous section, the energy consumption in a slot can be studied analytically for different PTC schemes.

We first consider the feasibility conditions under various PTC schemes. For any given PTC scheme, such condition requires that the transmission power of each node that is determined by the scheme must be positive. Observing (19), (28), and (34), it can be shown that the feasibility conditions for a node group under PTC-IPT, PTC-UT, and PTC-USG schemes are respectively given by the following:

$$
\mathbf{V}_{i} \text { is feasible iff }\left\{\begin{array}{l}
g_{i \Sigma}<1, \text { PTC-IPT } \\
g_{i 1}^{o}<\frac{B_{i 1} \gamma_{i 1}}{\sum_{j \in \mathbf{V}_{i}} B_{i j} \gamma_{i j}}, \text { PTC-UT } \\
R_{i}^{o}<\frac{W}{\delta \gamma_{i \Sigma}}, \text { PTC-USG }
\end{array}\right.
$$


where the subscript of each variable has been modified to point to the $i$ th group $\mathbf{V}_{i}$. In the iteration-based node scheduling, whenever a new node combination is under consideration, each node group in this combination will firstly be evaluated against (35) to decide their feasibility. Only those feasible node combinations will be processed by the heuristic algorithm described in Table 2.

The energy consumption in a slot under the three PTC schemes are derived as follows.

\subsubsection{PTC-IPT Scheme}

From (18), the optimal power index of node $i$ under PTC-IPT scheme is given by

$$
\begin{aligned}
g_{i}^{o(P T C-I P T)} & =\frac{\sqrt{\alpha_{c i r i} A_{i}}}{\sqrt{K}+\sum_{j=1}^{N} \sqrt{\alpha_{c i r j} A_{j}}} \\
& =\frac{\sqrt{\alpha_{c i r i} \delta B_{i} \gamma_{i}}}{\sqrt{\sum_{j=1}^{N} h_{j}^{-1} B_{j} \gamma_{j}}+\sum_{j=1}^{N} \sqrt{\alpha_{c i r j} B_{j} \delta \gamma_{j}}}
\end{aligned}
$$

Substituting (36), (19) and (20) into (4), and after some mathematical effort, the total energy consumption in a slot is given by

$$
E_{\text {total }}^{(P T C-I P T)}=\frac{1}{\eta W}\left(\sqrt{\sum_{i=1}^{N} h_{i}^{-1} B_{i} \gamma_{i}}+\sum_{i=1}^{N} \sqrt{\alpha_{c i r i} B_{i} \delta \gamma_{i}}\right)^{2} .
$$

\subsubsection{PTC-UT Scheme}

From (25), the optimal power index $g_{1}^{o}$ under PTC-UT scheme is given by

$$
\begin{aligned}
& g_{1}^{o(P T C-U T)}=\frac{\sqrt{E}}{\sqrt{C D}+\sqrt{E} D} \\
& =\frac{B_{1} \gamma_{1} \sqrt{\delta \sum_{i=1}^{N} \alpha_{c i r i}}}{\sqrt{\sum_{i=1}^{N} h_{i}^{-1} B_{i} \gamma_{i} \sum_{i=1}^{N} B_{i} \gamma_{i}}+\sqrt{\delta \sum_{i=1}^{N} \alpha_{c i r i}} \sum_{i=1}^{N} B_{i} \gamma_{i}} .
\end{aligned}
$$

Substituting (38) into (27), (28), and (4), and following a similar mathematical manipulation to the one used for the PTC-IPT scheme, the total energy consumption in a slot under PTC-UT is given by

$$
E_{\text {total }}^{(P T C-U T)}=\frac{1}{\eta W}\left(\sqrt{\sum_{i=1}^{N} h_{i}^{-1} B_{i} \gamma_{i}}+\sqrt{\delta \sum_{i=1}^{N} \alpha_{\text {ciri }}} \sqrt{\sum_{i=1}^{N} B_{i} \gamma_{i}}\right)^{2} .
$$

\subsubsection{PTC-USG Scheme}


From (31), the optimal transmission rate under PTC-USG scheme is given by

$$
\begin{aligned}
& R^{o(P T C-U S G)}=\frac{\sqrt{H}}{\sqrt{F G}+\sqrt{H} G} \\
& =\frac{W \sqrt{\sum_{i=1}^{N} \alpha_{c i r i} B_{i}}}{\sqrt{\sum_{i=1}^{N} h_{i}^{-1} B_{i} \gamma_{i}} \sqrt{\delta \sum_{i=1}^{N} \gamma_{i}}+\sqrt{\sum_{i=1}^{N} \alpha_{c i r i} B_{i}} \delta \sum_{i=1}^{N} \gamma_{i}} .
\end{aligned}
$$

Substituting (40) into (33), (34), and (4), the total energy consumption in a slot under PTCUSG scheme is given by

$$
E_{\text {total }}^{(P T C-U S G)}=\frac{1}{\eta W}\left(\sqrt{\sum_{i=1}^{N} h_{i}^{-1} B_{i} \gamma_{i}}+\sqrt{\sum_{i=1}^{N} \alpha_{c i r i} B_{i}} \sqrt{\delta \sum_{i=1}^{N} \gamma_{i}}\right)^{2}
$$

\subsubsection{Comparison of Energy Consumption}

In order to compare the energy efficiency of different schemes, we assume for simplicity a homogeneous WSN, i.e., $\alpha_{\text {ciri }}=\alpha_{\text {cir }}$ and $\gamma_{i}=\gamma$ for all $i$. With this assumption, the energy consumption under various schemes can be further simplified as

$$
E_{\text {total }}^{(P T C-I P T)}=\frac{1}{\eta W}\left(\sqrt{\sum_{i=1}^{N} h_{i}^{-1} B_{i} \gamma}+\sqrt{\alpha_{c i r} \delta \gamma} \sum_{i=1}^{N} \sqrt{B_{i}}\right)^{2}
$$

and

$$
\begin{aligned}
& E_{\text {total }}^{(P T C-U T)}=E_{\text {total }}^{(P T C-U S G)} \\
& =\frac{1}{\eta W}\left(\sqrt{\sum_{i=1}^{N} h_{i}^{-1} B_{i} \gamma}+\sqrt{\alpha_{c i r} \delta \gamma N} \sqrt{\sum_{i=1}^{N} B_{i}}\right)^{2} .
\end{aligned}
$$

The only difference between (42) and (43) is the second term of the base. Because $\sqrt{x}$ is a concave function, according to Jensen's inequality, we have

$$
\sqrt{\frac{1}{N} \sum_{i=1}^{N} B_{i}} \geq \frac{1}{N} \sum_{i=1}^{N} \sqrt{B_{i}}
$$

or equivalently $\sqrt{N} \sqrt{\sum_{i=1}^{N} B_{i}} \geq \sum_{i=1}^{N} \sqrt{B_{i}}$. Therefore, $E_{\text {total }}^{P T C-U T}=E_{\text {total }}^{P T C-U S G} \geq E_{\text {total }}^{(P T C-I P T)}$, which is in accordance with the intuition that PTC-IPT should be more energy-efficient because of its larger degree of control.

An observation of the two terms of the base of (42) and (43) shows that the total energy consumption is likely to be dominated by the first term because of the relatively large value of 
the inverse channel gain $h_{i}^{-1}$. Becase (42) and (43) have the same first term in their base, we can expect that although PTC-IPT has the complete freedom to control both the transmit power and transmission time of every sensor node, it may not bring significant efficiency improvement over PTC-UT and PTC-USG. We will verify this expectation later in numerical examples.

\subsubsection{Comparison of Computation Overhead}

PTC-UT and PTC-USG are easier to implement than PTC-IPT. The number of control variables in PTC-UT and PTC-USG is approximately half of that in PTC-IPT scheme. In addition, as shown in [28], under PTC-IPT scheme an iterative algorithm need to be executed at $\mathrm{CH}$ to solve for the optimal transmission power and time of each node. In contrast to this centralized operation, the optimization under PTC-UT and PTC-USG schemes can be realized distributedly at each node with some assistance from node $o$. Specifically, given the values of

$g_{1}^{o}, B_{1} \gamma_{1}$, and $\sum_{i=1}^{N} B_{i} \gamma_{i}$, the optimal transmit power and time under the PTC-UT scheme can be computed locally by each node according to (27) and (28). Because the required information is the same for all nodes, node $o$ simply needs to broadcast them throughout the system. Similarly, (33) and (34) shows that under the PTC-USG scheme, broadcasting the values of $R^{o}$ and $\sum_{i=1}^{N} \gamma_{i}$ is sufficient for local computation of the optimal transmission power and time at individual nodes. As a result, PTC-UT and PTC-USG have much smaller computing complexity than that of PTC-IPT scheme.

\section{Numerical Examples}

In this section, through numerical experiments, we verify the performance of the proposed PTC policies and the heuristic scheduling algorithm. Because the STDMA algorithm at the network level has assigned interfering clusters into different frames, it is sufficient for us to only consider a single-cluster system in our simulation. All numerical experiments presented below were conducted using MATLAB.

\subsection{Simulation Setup}

We consider a 20 meter $\times 20$ meter square field, as shown in Figure 3, over which $N$ homogeneous SNs are distributed uniformly. All SNs on this field are organized into one cluster and the $\mathrm{CH} o$ (in the single-cluster case, this is also the sink) is located at $(D, 0)$. A frame contains $M$ slots, 


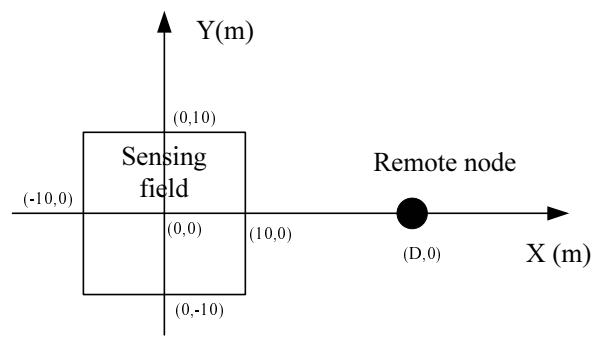

Figure 3: Sensing field used in the numerical examples.

each of which is of length $\frac{1}{M}$ seconds. In our simulation, we only consider the case of uplink (from $\mathrm{SN}$ to $\mathrm{CH}$ ) communication, therefore all $M$ slots in a frame are dedicated for the uplink transmission. In addition, we do not simulate the CAR packet transmission process in the access window because such process is of no difference from the well-known CSMA communication. Because of the short length of CAR packets, in our simulation we simply assume all active SNs in a frame can send their CAR successfully, thus will be admitted by the CH properly.

For each node, we use the following parameters. The power amplifier energy efficiency is $\eta=0.9$. A rectangular spreading chip is assumed, i.e. $\delta=\frac{2}{3}$. The threshold of the received SINR is 4 for all SNs. The spread spectrum bandwidth is $W=1 \mathrm{MHz}$ and the single-sided power spectrum density of the AWGN is $N_{0}=10^{-15} \mathrm{~W} / \mathrm{Hz}$. The channel gain from node $i$ to the $\mathrm{CH}$ is given by

$$
h_{i}=L\left(d_{0}\right)\left(\frac{d_{i}}{d_{0}}\right)^{-\mu} Y_{i}\left(X_{I i}^{2}+X_{Q i}^{2}\right),
$$

where $L\left(d_{0}\right)=\frac{G_{t} G_{r} \lambda^{2}}{16 \pi^{2} d_{0}^{2}}$ is the path loss of the close-in distance $d_{0}, G_{t}$ and $G_{r}$ are the antenna gains of the transmitter and the receiver, respectively, and $\lambda$ is the wavelength of the carrier. We set $d_{0}=10$ meters, $G_{t}=G_{r}=1$, and assume a carrier frequency of $2.4 \mathrm{GHz}$. The parameter $d_{i}$ is the distance between node $i$ and the remote node. The $Y_{i}$ 's are i.i.d. lognormal shadowing with a standard deviation of $7 \mathrm{~dB}$. Moreover, $X_{I i}$ and $X_{Q i}$ are the real and the imaginary parts of a Rayleigh fading channel gain and follow a Gaussian distribution with mean zero and variance $\frac{1}{2}$. $\mu$ is the path loss exponent and is assumed to be 2 in our system.

\subsection{Results for CDMA Transmission}

In this scenario, we set $M=1$ and compare the performance of the proposed PTC policies for the CDMA transmissions. To get an insight into the various schemes, we first consider a small example consisting of 5 sensor nodes, whose parameters and optimization results are given in Table 3. 


\begin{tabular}{|c|c|c|c|c|c|c|c|c|c|c|c|c|}
\hline \multirow[t]{2}{*}{ node } & \multirow[t]{2}{*}{$h_{i}\left(\times 10^{-6}\right)$} & \multirow[t]{2}{*}{$B_{i}$ (bits) } & \multicolumn{2}{|c|}{ PTC-IPT (GP) } & \multicolumn{2}{|c|}{ PTC-IPT } & \multicolumn{2}{|c|}{ PTC-UT } & \multicolumn{2}{|c|}{ PTC-USG } & \multicolumn{2}{|l|}{ MDT } \\
\hline & & & $P_{t i}^{o}$ & $T_{i}^{o}$ & $P_{t i}^{o}$ & $T_{i}^{o}$ & $P_{t i}^{o}$ & $T_{i}^{o}$ & $P_{t i}^{o}$ & $T_{i}^{O}$ & $P_{t i}^{o}$ & $T_{i}^{O}$ \\
\hline 1 & 0.0634 & 98 & 2.319 & 3.8 & 2.315 & 3.8 & 2.224 & 4.2 & 2.410 & 3.9 & 0.00619 & 1000 \\
\hline 2 & 0.0068 & 124 & 24.326 & 4.2 & 24.113 & 4.2 & 26.056 & 4.2 & 22.315 & 4.9 & 0.0725 & 1000 \\
\hline 3 & 0.029 & 86 & 4.75 & 3.5 & 4.743 & 3.5 & 4.269 & 4.2 & 5.271 & 3.4 & 0.01188 & 1000 \\
\hline 4 & 0.8816 & 112 & 0.178 & 4.0 & 0.178 & 4.0 & 0.183 & 4.2 & 0.173 & 4.4 & $5.1 \times 10^{-4}$ & 1000 \\
\hline 5 & 0.0029 & 111 & 53.967 & 4.0 & 54.327 & 4.0 & 55.541 & 4.2 & 53.140 & 4.4 & 0.1545 & 1000 \\
\hline$E_{\text {total }}^{o}$ & $\begin{array}{lll}- & - \\
\end{array}$ & - & & & 577.6 & & 621.5 & & 621.3 & & $5.027 \times$ & \\
\hline
\end{tabular}

Table 3: Parameters and optimization results for a 5-node CDMA WSN, where the units of power, time, and energy are $\mathrm{mW}, \mathrm{ms}$, and $\mu \mathrm{J}$, respectively $\left(\alpha=10 \mathrm{~mW}, P_{\max }=100 \mathrm{~mW}\right)$.

Three observations can be made from Table 3. First, under PTC-IPT scheme, sufficient accuracy is achieved by the approximated solution in [28]. This is observed by comparing the approximated solutions (columns 6 and 7) with the results (columns 4 and 5) computed from the GP-based numerical algorithm [28]. It shows that there is almost no difference between these two results. Second, significant energy savings can be achieved by all three PTC schemes over those "optimal" schemes proposed in the context of cellular networks. This is demonstrated by comparing the results from all three PTC schemes with that of the maximum delay transmission (MDT) scheme in Table 3. The MDT scheme always assigns the longest possible transmission time (i.e., the frame length) to each node and calculates the optimal transmission power by using (12). When circuit energy consumption is negligible, this is the optimal transmission control scheme that minimizes the total transmission energy [12]. However, in a WSN where the circuit energy is non-negligible, MDT scheme losses its energy superiority because the transmission energy savings achieved by prolonging transmission durations are out weighted by the excessive circuit energy consumption. In contrast, the various PTC schemes proposed in this work can save significant energy by explicitly taking the transmission-circuit energy tradeoff into consideration. Third, it is further noted that while PTC-IPT involves nearly twice as many control variables as PTC-UT and PTC-USG, it achieves only minor efficiency improvement (around 7.6\%).

To compare the energy efficiencies of the various PTC policies in a more practical scenario, we further simulate a larger network configuration consisting of much more nodes. We plot in Figures 4 and 5 the bit energy efficiencies under different schemes as a function of network size and the circuit power consumption, respectively. The results in these figures are based on averaging over 1000 simulation cycles. In each cycle, the channel gain of every link is varied 


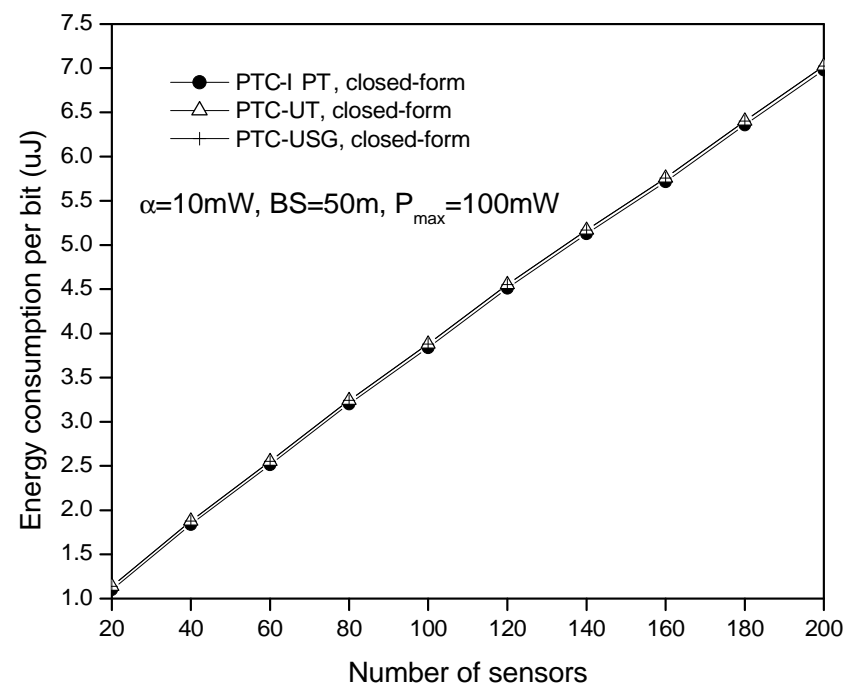

Figure 4: Bit energy efficiency vs. number of sensors.

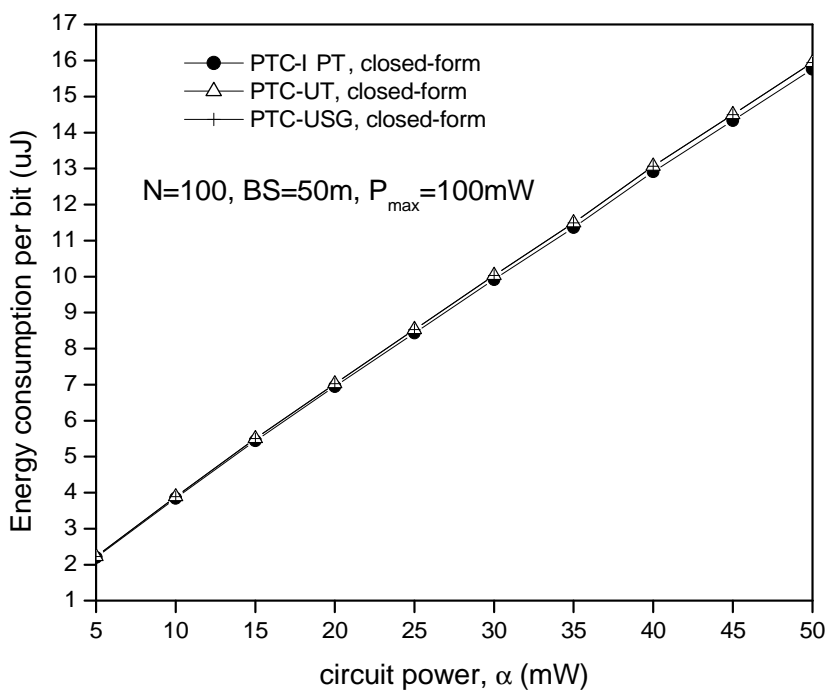

Figure 5: Bit energy efficiency vs. circuit power. 
according to (45) and the traffic transmitted by different nodes follows independently and identically distributed (i.i.d.) Poisson of mean 100 bits. From Figures 4 and 5, it is shown that only a marginal energy efficiency improvement (say, about 1\%) is achieved by PTC-IPT over PTC-UT and PTC-USG. This confirms our expectation when we compare (42) and (43) in the previous section. The observation that the energy efficiency is insensitive to the transmission time control (or equivalently, the transmission rate control) when the transmission power control has been performed, re-confirms and enhances the conclusion made in [30]. In that work, it has been found that for the single-link case, rate control can only contribute marginal improvement to the link efficiency when power control has been conducted. The significance of our work is that it extends the validity of this conclusion to multiple access systems and to the scenario that not only the communication energy, but also the circuit energy consumption are accounted for in the performance metrics.

\subsection{Results for Hybrid TDMA/CDMA Transmission}

In this experiment, we are interested in the extra energy savings brought by scheduling the transmissions of active SNs into multiple slots in a frame. We are also interested in the accuracy of the heuristic algorithms for searching the optimal schedule. In order to make our results repeatable, we use the following deterministic data set in this example: we consider a cluster which includes the five SNs listed in Table 3 and other 4 additional SNs, whose channel/traffic parameters $U_{i}=\left(h_{i}, B_{i}\right)$ are given by $U_{6}=\left(0.334 \times 10^{-6}, 72\right), U_{7}=\left(0.0722 \times 10^{-6}, 85\right)$, $U_{8}=\left(0.0035 \times 10^{-6}, 120\right)$, and $U_{9}=\left(0.00581 \times 10^{-6}, 91\right)$, respectively. The settings $(N=9, M$ ranges from 1 to 7 ) here is comparable to that of a GTS cluster in an IEEE 802.15.4 WPAN, where $N \leq 7$ and $M \leq 7$. The channel and traffic parameters of all the nodes are randomly generated according to (45) and a Poisson distribution with mean of 100 bits, respectively.

The total energy consumption is plotted as function of number of slots in Figure 6, for which the heuristic algorithm is used for scheduling and PTC-USG is used for the CDMA transmission in each slot. To demonstrate the energy advantages of this heuristic algorithm, we also plot in Figure 6 the energy consumptions when a load-balancing scheduler is used. This algorithm schedules a node to the slot which has the minimum traffic. When $M=1$, the system is a pure CDMA network where all active nodes transmit simultaneously (there is no scheduling), leading to high interference. As a result, the energy efficiency of the system is poor. With the $\underline{\text { increase of } M \text {, less number of nodes will transmit simultaneously, making the interference in }}$ 


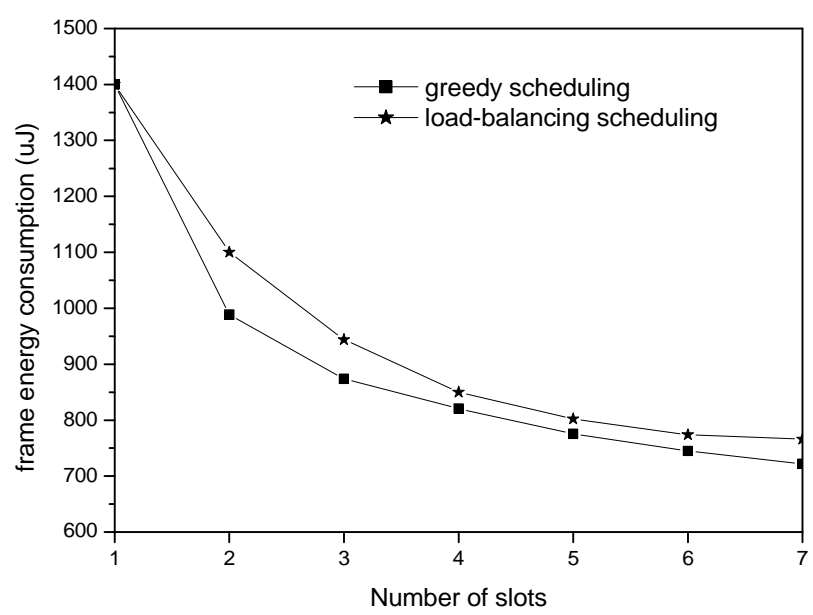

Figure 6: Energy consumption vs. number of slots.

each slot smaller. So the effect of scheduling on system's energy efficiency becomes more and more observable. It is clear that significant energy efficiency improvement, e.g., around $100 \%$ when 7 slots are used, can be obtained by combining TDMA with CDMA transmission. In addition, the heuristic algorithm always has an energy gain over the load-balancing algorithm. Especially, when $M$ is small, say, $M=2$ or 3 , the greedy algorithm can save more than $10 \%$ energy compared with the load-balancing scheduling. This finding indicates that the greedy algorithm is more effective in reducing MAI for simultaneous transmissions.

In Tables 4 and 5, we contrast the schedules determined by the heuristic algorithm to the optimal ones found by using DES. To demonstrate the energy savings, we also list the results from the load-balancing scheduler. This comparisons are conducted under various values of $N$ and $M$. In each comparison, we suppose only nodes 1 to $\mathrm{N}$ are transmitted in the frame. Due to the limitation of computation capability, the largest $N$ and $M$ that we have verified are $N=9$ and $M=3$.

\begin{tabular}{|c|c|c|c|c|c|c|c|c|c|}
\hline \multirow{2}{*}{$N$} & \multicolumn{3}{|c|}{ Greedy Algorithm } & \multicolumn{3}{c|}{ Exhaustive Search } & \multicolumn{3}{c|}{ Load-Balancing } \\
\cline { 2 - 10 } & $E_{\text {total }}(\mu J)$ & slot 1 & slot 2 & $E_{\text {total }}(\mu J)$ & slot 1 & slot 2 & $E_{\text {total }}(\mu J)$ & slot 1 & slot 2 \\
\hline 5 & 464.45 & 2,5 & $1,3,4$ & 464.45 & 2,5 & $1,3,4$ & 506 & $1,3,5$ & 2,4 \\
\hline 6 & 513.35 & $2,3,5$ & $1,4,6$ & 495 & $1,3,4,6$ & 2,5 & 545.25 & $1,3,5$ & $2,4,6$ \\
\hline 7 & 587.54 & $2,3,5,7$ & $1,4,6$ & 543.67 & $1,3,4,6,7$ & 2,5 & 611.44 & $1,3,5,7$ & $2,4,6$ \\
\hline 8 & 813.85 & $2,5,8$ & $1,3,4,6,7$ & 813.85 & $2,5,8$ & $1,3,4,6,7$ & 901.8 & $1,3,5,7$ & $2,4,6,8$ \\
\hline 9 & 988.62 & $2,5,8,9$, & $1,3,4,6,7$ & 988.62 & $2,5,8,9$ & $1,3,4,6,7$ & 1100 & $1,3,5,7,9$ & $2,4,6,8$ \\
\hline
\end{tabular}

Table 4: Schedule comparison for various values of $\mathrm{N}(\mathrm{M}=2)$.

An observation on Tables 4-5 shows that, in addition to a near $10 \%$ energy saving over load- 


\begin{tabular}{|c|c|c|c|c|c|c|c|c|c|c|c|c|}
\hline \multirow[t]{2}{*}{$N$} & \multicolumn{4}{|c|}{ Greedy Algorithm } & \multicolumn{4}{|c|}{ Exhaustive Search } & \multicolumn{4}{|c|}{ Load-Balancing } \\
\hline & $E_{\text {total }}(\mu J)$ & slot 1 & slot 2 & slot 3 & $E_{\text {total }}(\mu J)$ & slot 1 & slot 2 & slot 3 & $E_{\text {total }}(\mu J)$ & slot 1 & slot 2 & slot 3 \\
\hline 5 & 423.58 & 5 & $1,3,4$ & 2 & 423.58 & 5 & $1,3,4$ & 2 & 442.51 & 1,5 & 2 & 3,4 \\
\hline 6 & 446.88 & 5 & $1,4,6$ & 2,3 & 446.88 & $1,4,6$ & 2,3 & 5 & 476.44 & 1,5 & 2,6 & 3,4 \\
\hline 7 & 486.28 & $4,6,7$ & 1,3 & 2,5 & 486.28 & $4,6,7$ & 1,3 & 2,5 & 523.72 & 1,5 & $2,6,7$ & 3,4 \\
\hline 8 & 730.3 & 5,8 & $1,4,6,7$ & 2,3 & 730.33 & 5,8 & $1,4,6,7$ & 2,3 & 797 & 1,5 & $2,6,7$ & $3,4,8$ \\
\hline 9 & 873.93 & 2,9 & $1,3,4,6,7$ & 5,8 & 873.93 & 5,8 & $1,3,4,6,7$ & 2,9 & 945 & $1,5,9$ & $2,6,7$ & $3,4,8$ \\
\hline
\end{tabular}

Table 5: Schedule comparison for various values of $\mathrm{N}(\mathrm{M}=3)$.

balancing scheme, the heuristic algorithm in fact gives the optimal schedules in most cases. For those rare exceptions, i.e., $(N=6, M=2)$ and $(N=7, M=2)$, the error on the energy is less than $8 \%$. Our findings indicate that for a system with moderate numbers of nodes and slots, the greedy algorithm can give at least near-optimal (or even optimal, in the shown cases) schedules.

\section{Conclusions}

In this paper, we proposed a hybrid TDMA/CDMA mechanism to improve the scalability and flexibility of the pure TDMA protocol while maintaining high energy efficiency. Both the communication and the circuity energy consumptions are accounted for in the problem formulation. The improvement of the energy efficiency is conducted in three different levels. At the network level, the interfering clusters are assigned into different frame to eliminate inter-cluster interference. At the cluster level, the optimal scheduling of nodes' transmission is decided using iterative method; At the slot level, the optimal PTC for concurrent CDMA transmission is decided based on some optimization formulations.

In correspondence to the three-level improvement, the major contributions of this paper are threefold. First, a vertex-coloring problem, whose solutions have been well known, was proposed to model the inter-cluster interference-control problem. Second, a heuristic algorithm that has polynomial computing complexity was proposed to solve the optimal scheduling problem at the cluster level. In most cases of our numerical examples, this heuristic algorithm actually provided the optimal schedules. Third, at the slot level, regarding the joint transmission power/time control, we extend our optimization work in [28] by proposing two simplified power/time control (PTC) schemes. Compared with the original centralized PTC algorithm in [28], the new schemes can be implemented in a distributed way. They cut the number of 
control variables by almost $50 \%$ while still achieving above $90 \%$ of the energy efficiency provided by the old algorithm. Our work clearly demonstrated the insensitivity of the energy efficiency to the transmission time control (or equivalently, the transmission rate control) when the transmission power control has been performed. This feature re-confirms and enhances a well-known observation that originally applies to the single-link case, i.e., rate control only contribute marginal improvement to the link efficiency when power control has been conducted. The significance of our work is to extend the validity of this observation to multiple access systems and to the scenario that not only the communication energy, but also the circuit energy consumption are accounted for in the performance metrics. In our current formulation, the boundary between the up-link and down-link sub-frames is fixed, and the number of slots in a $\underline{\text { sub-frame is constant. Our future work will consider a more flexible setup, where this boundary }}$ is adjustable according to the traffic loads over the up-link and down-link. In this case, the number of slots in each sub-frame is a variable to be optimized.

\section{References}

[1] I. F. Akyildiz, W. Su, Y. Sankarasubramaniam, and E. Cayirci, "A survey on sensor networks," IEEE Communications Magazine, pp. 102-114, Aug. 2002.

[2] W. Heinzelman, A. Chandrakasan, and H. Balakrishnan, "An application-specific protocol architecture for wireless microsensor networks," IEEE Transactions on Wireless Communications, vol. 1, no. 4, pp. 660-670, Oct. 2002.

[3] W. Ye, J. Heidemann, and D. Estrin, "An energy-efficient MAC protocol for wireless sensor networks," Proc. of the IEEE INFOCOM 2002 Conference, vol. 3, June 2002, pp. 1567-1576.

[4] T. V. Dam and K. Langendoen, "An adaptive energy-efficient MAC protocol for wireless sensor networks," Proc. of the First ACM Conference on Embedded Networked Sensor Systems (SenSys), Los Angeles, Nov. 2003, pp. 171-180.

[5] T. Zheng, S. Radhakrishnan, and V. Sarangan, "PMAC: An adaptive energy-efficient MAC protocol for wireless sensor networks", Proc. of IPDPS'05, Apr. 2005.

[6] I. Rhee, A. Warrier, M. Aia, J. Min, and M. L. Sichitiu, "Z-MAC: A hybrid MAC for wireless sensor networks," IEEE/ACM Transactions on Networking, vol. 16, no. 3, June 2008, pp. 511-524.

[7] Y. E. Sagduyu and A. Ephremides, "The problem of medium access control in wireless sensor networks," IEEE Wireless Communications, Dec. 2004, pp. 44-53.

[8] IEEE standards 802.15.1: Wireless Medium Access Control (MAC) and Physical Layer (PHY) Specifications for Wireless Personal Area Networks (WPANs).

[9] IEEE standards 802.15.4: Wireless medium access control (MAC) and physical layer (PHY) specifications for low-rate wireless personal area networks (LR-WPANS).

[10] Y. Chen and E. Fleury, "A distributed policy scheduling for wireless sensor networks," Proc. of the IEEE INFOCOM 2007 Conference, pp. 1559-1567, 2007. 
[11] S. De, C. Qiao, D. A. Pados, M. Chatterjee, and S. J. Philip, "An integrated cross-layer study of wireless CDMA sensor networks," IEEE Journal on selected areas in communictions, vol. 22, no. 7 , Sep. 2004, pp. 1271-1285.

[12] R. Berry and R. Gallager, "Communication over fading channels with delay constraints," IEEE Trans. on Inform. Theory, vol. 48, no. 5, pp. 1135-1149, May 2002.

[13] J. Li and G. Y. Lazarou, "A bit-map-assisted energy-efficient MAC scheme for wireless sensor networks," Proc. of ACM IPSN'04, Apr. 2004, Berkeley, CA, USA.

[14] S. Cui, A. J. Goldsmith, and A. Bahai, "Joint modulation and multiple access optimization under energy constraints," Proc. of IEEE Globecom'04, Nov 2004, vol. 1, pp. 151-155.

[15] S. Cui, R. Madan, A. J. Goldsmith, and S. Lall, "Cross-layer energy and delay optimization in small-scale sensor networks," IEEE Trans. on Wireless Communications, vol. 6, no. 10, pp.36883699, Oct. 2007.

[16] H. Haas and S. McLaughlin, "A dynamic channel assignment algorithm for a hybrid TDMA/CDMA-TDD interface using the novel TS-Opposing technique," IEEE Journal on Selected Areas in Communications, vol. 19, no. 10, pp. 1831-1846, Oct. 2001.

[17] T. ElBatt and A. Ephremides, "Joint scheduling and power control for wireless ad-hoc networks," Proc. of the INFOCOM 2002 Conference, vol. 2, pp. 976-984, June 2002.

[18] S. Suh and D. J. Goodman, "Throughput maximization in multimedia TDMA/CDMA systems," Proc. of IEEE VTC'05 Fall, vol. 2, pp. 730-736, Sept. 2005.

[19] L. Ortigoza-Guerrero and A. H. Aghvami, "A distributed dynamic resource allocation for a hybrid TDMA/CDMA system," IEEE Transactions on Vehicular Technology, vol. 47, no. 4, pp. 1162-1178, Nov. 1998.

[20] G. Pennett, and C. Dion-Schwarz, "QoS and scheduling based schemes for a generalized hybrid request TDMA/CDMA protocol for wireless multimedia," Proc. of the First International Conference on Quality of Service in Heterogeneous Wired/Wireless Networks, pp. 325-328, 2004.

[21] F. Wang, M. Krunz, and S. Cui, "Price-based spectrum management in cognitive radio networks," IEEE Journal of Selected Topics in Signal Processing, vol. 2, no. 1, Feb. 2008, pp. 74-87.

[22] F. Casoni, G. Immovilli, and M. L. Merani, "Integrating voice and data applications in T/CDMA architectures," IEEE Communication Letters, vol. 5, no. 9, pp. 378-380, Sept. 2001.

[23] R. Nelson and L. Kleinrock, "Spatial TDMA: a collision-free multihop channel access protocol," IEEE Transactions on Communications, vol. 33, no. 9, pp. 934-944, Sept. 1985.

[24] O. Younis and S. Fahmy, "Distributed clustering in ad-hoc sensor networks: A hybrid, energyefficient approach," Proc. of the IEEE INFOCOM 2004 Conference, Hong Kong, Mar. 2004. An extended version appeared in IEEE Transactions on Mobile Computing, vol. 3, no. 4, pp. 366-379, Oct-Dec 2004.

[25] A. D. Amis, R. Prakash, T. H. P. Vuong, and D. T. Huynh, "Max-min D-cluster formation in wireless ad hoc networks," Proc. of the IEEE INFOCOM 2000 Conference, pp. 32-41, Mar. 2000.

[26] S. Cui, A. J. Goldsmith, and A. Bahai, "Energy-constrained modulation optimization," IEEE Transactions on Wireless Communications, vol. 4, no. 5, pp. 2349-2360, Sep. 2005.

[27] S. Ramanathan and E. L. Lloyd, "Scheduling algorithms for multihop radio networks," IEEE/ACM Transactions on Networking, vol. 1, no. 2, Apr. 1993, pp. 166-177.

[28] T. Shu, M. Krunz, and S. Vrudhula, "Joint optimization of transmit power-time and bit energy efficiency in CDMA wireless sensor networks," IEEE Trans. on Wireless Communication, vol. 5, no. 11, pp. 3109-3118, Nov. 2006. 
[29] S. Cui, J. Xiao, A. J. Goldsmith, Z. Q. Luo, and H. V. Poor, "Estimation diversity and energy efficiency in distributed sensing," IEEE Transactions on Signal Processing, vol. 55, no. 9, pp. 4683-4695, Sep. 2007.

[30] S. T. Chung and A. J. Goldsmith, "Degrees of freedom in adaptive modulation: a unified view," IEEE Transactions on Communications, vol. 49, no. 9, Sep. 2001, pp. 1561-1571.

[31] J. Pan, Y. T. Hou, L. Cai, Y. Shi, and S. X. Shen, "Topology control for wireless sensor networks," Proc. of ACM MobiCom 2003, pp. 286-299.

[32] K. Romer and F. Mattern, "The design space of wireless sensor networks," IEEE Wireless Communications, vol. 11, no. 6, pp. 54-61, Dec. 2004.

[33] S. Boyd and L. Vandenberghe, Convex Optimization, Cambridge Univ. Press, Cambridge, U.K., 2004.

[34] K. Hwang, J. In, N. Park, and D. Eom, "A design and implementation of wireless sensor gateway for efficient querying and managing through world wide web," IEEE Transactions on Consumer Electronics, Vol. 49, No. 4, pp. 1090-1097, Nov. 2003.

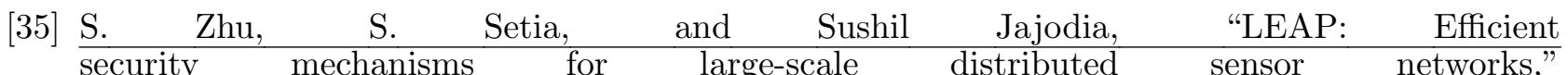
Proc. of the 10th ACM Conference on Computer and Communications Security, $\quad$ pp. $\quad 62-72$, 2003.

[36] Q. Fang, J. Gao, L. J. Guibas, V. de Silva, and L. Zhang, "GLIDER: Gradient landmark-based distributed routing for sensor networks," Proc. of the IEEE INFOCOM 2005 Conference, pp. 339-350. 